\title{
In silico modeling of the pore region of a KCNQ4 missense mutant from a patient with hearing loss
}

Kazunori Namba ${ }^{1 \dagger}$, Hideki Mutai ${ }^{1 \dagger}$, Hiroki Kaneko ${ }^{2}$, Sho Hashimoto ${ }^{3}$ and Tatsuo Matsunaga ${ }^{1 *}$

\begin{abstract}
Background: Mutation of the voltage-gated potassium channel KCNQ4 causes DFNA2-type nonsyndromic autosomal dominant sensorineural hearing loss. KCNQ4 is expressed predominantly in the auditory sensory outer hair cells, which are critical for sound amplification.

Results: We sequenced KCNQ4 from Japanese patients with sensorineural hearing loss, and identified a novel missense mutation encoding a Tyr270His located at the $\mathrm{N}$-terminus of the highly conserved pore helix sequence. As this patient was not accessible to us and information about them was limited, we used molecular modeling to investigate whether this novel mutation is hypothetically pathogenic. A careful examination of an in silico structural model of the KCNQ4 pore region revealed that the Tyr270His mutation caused an alteration in the electrostatic surface potential of the pore helix.
\end{abstract}

Conclusion: We propose two possible means by which the Tyr270His mutation causes hearing loss: a positively charged His270 side chain might enhance the helix dipole moment of the pore helix, thereby destabilizing the helix and/or the pore region, or it might disturb transport of $\mathrm{K}^{+}$through the channel by electrostatic repulsion.

Keywords: Channel, Deafness, Electrostatic, Hearing loss, Molecular Modeling, Mutation, Potassium, Structure

\section{Background}

Deafness is a frequently inherited sensory disorder. For every 1000 newborns, more than $1 \%$ have bilateral sensorineural hearing loss (SNHL), and $50-70 \%$ of the cases are monogenic disorders $[1,2]$. Hereditary hearing loss is classified as syndromic and nonsyndromic [3]. The genetic causes of nonsyndromic hearing loss are autosomal dominant, autosomal recessive, $\mathrm{X}$-chromosome linked, and mitochondrial in nature. To date, 25, 40,3, and 6 genes have been identified that are responsible for autosomal dominant-, autosomal recessive-, X-chromosome linked-, and mitochondrial hearing loss, respectively http://hereditaryhearingloss.org/. In particular, autosomal dominant nonsyndromic sensorineural deafness type 2 (DFNA2) hearing loss affects the ability of children to hear high frequencies, and results in hearing loss at all frequencies later in life [4]. KCNQ4 is the causative gene [5] and encodes the membrane protein KQT-like, subfamily member 4 ,

\footnotetext{
* Correspondence: matsunagatatsuo@kankakuki.go.jp

† Contributed equally

'Laboratory of Auditory Disorders, National Institute of Sensory Organs,

National Tokyo Medical Center, Tokyo, Japan

Full list of author information is available at the end of the article
}

which contains 695 amino acid residues in its longest isoform.

KCNQ4 is a member of the five muscarinic receptorregulated and voltage-gated potassium (M-type $\mathrm{K}^{+}$channel) channels present in ear and epithelia [6]. In the inner ear, KCNQ4 subunits assemble to form homomeric channels that help with the recycling of potassium [5]. Because patients with $K C N Q 4$ mutations show progressive hearing loss, development of drugs that improve KCNQ4 function should attenuate hearing loss.

While conducting an ongoing genetic study of hereditary hearing loss, we identified a mutated $K C N Q 4$ from a Japanese patient in which the encoded Tyr270 residue was replaced with His. Structural analysis of mutations using molecular modeling is a practical technology to investigate their pathogenicity in patients who are not readily available for analysis. To determine how the mutated protein (denoted Tyr270His) induced hearing loss, we built models of the KCNQ4 and Tyr270His pore regions. By examining models that included electrostatic potentials, we were able to propose two molecular mechanisms that could have caused the patient's hearing loss.

\section{() Biomed Central}




\section{Results and discussion}

Prior to this report, 14 missense mutations, a splice-site mutation, and 2 small deletion mutations in KCNQ4 were reported to be associated with hearing loss [7-9]. Because 11 of the missense mutations were found in the pore region, it was considered a pathogenic "hot spot". We therefore sequenced KCNQ4 exons 5, 6, and 7 (which encode the pore region) of Japanese patients with bilateral nonsyndromic hearing loss. A new heterozygotically presented $K C N Q 4$ missense mutation was found at position 808 , in which the wild-type thymine (T) was replaced by cytosine $(\mathrm{C})$. This results in a Tyr270His mutation in the protein (Figure 1).

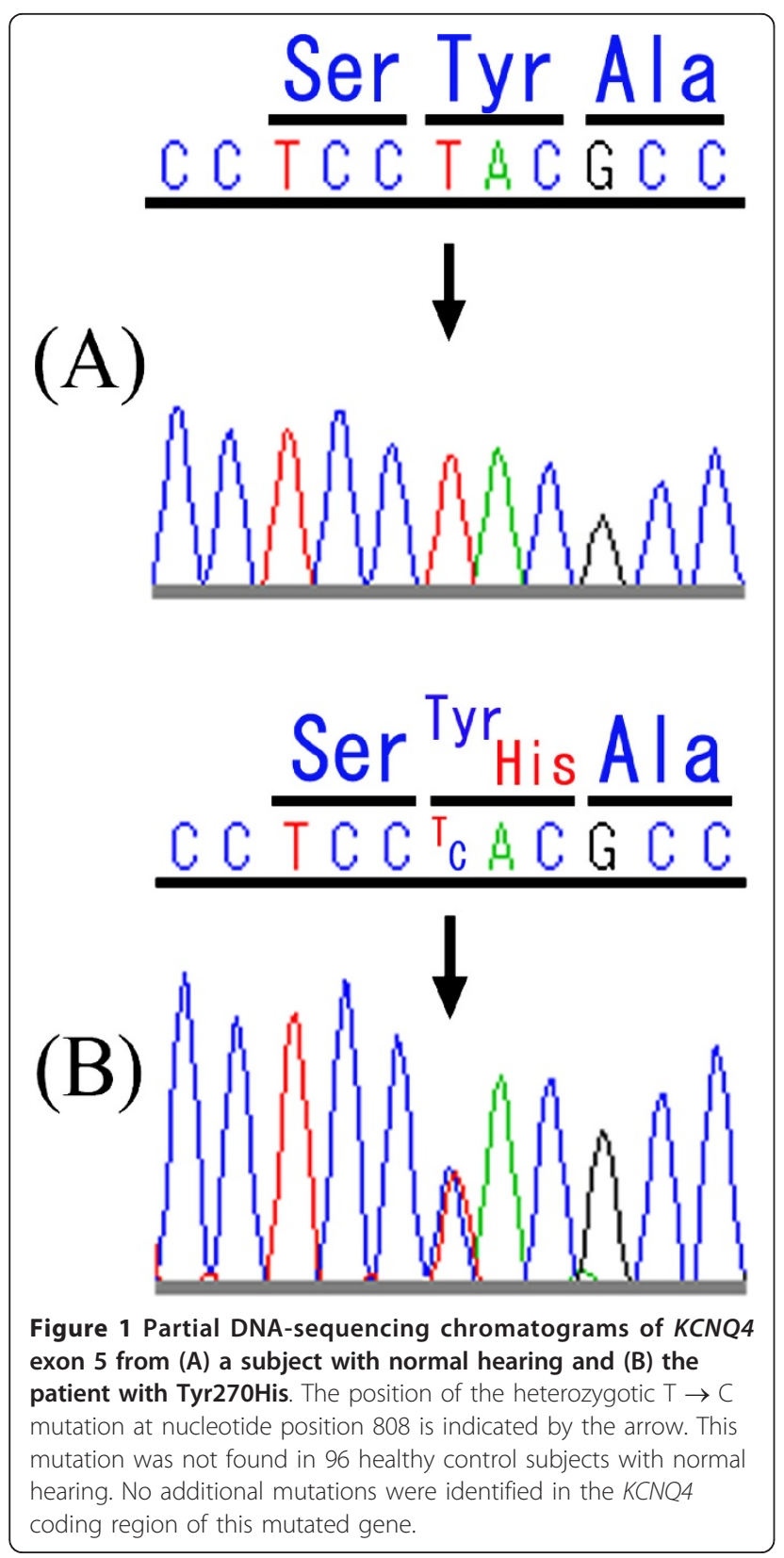

The patient who carried the Tyr270His mutation was a 28-year-old female with congenital, progressive hearing loss. An audiometric examination found mild hearing loss in her left ear and moderate hearing loss in her right ear, with steeply sloping audiometric configurations in both ears (Figure 2). The patient had no inner ear malformations, according to computational tomography. None of her family members had hearing loss, and no environmental factors that might have caused the hearing loss were reported (e.g., exposure to noise or ototoxic drugs.) DNA samples were not obtained from her family.

Similar to other voltage-gated $\mathrm{K}^{+}(\mathrm{Kv})$ channels, $\mathrm{KCNQ} 4$ probably contains six transmembrane $\alpha$-helices, (S1-S6), a pore helix $(\mathrm{PH})$, a pore-loop (P-loop), a short $\mathrm{N}$-terminal region, and a long $\mathrm{C}$-terminal region (Figure $3 \mathrm{~A})$. The pore regions of $\mathrm{Kv}$ channels are formed by the contiguous regions S5, PH, P-loop, and S6. Functional and structural characterizations of $\mathrm{Kv}$ channels have demonstrated that the $\mathrm{PH}$ and $\mathrm{P}$-loop are responsible for selective $\mathrm{K}^{+}$transport, whereas $\mathrm{S} 4$, acting as a voltage sensor, regulates the open/closed state of the channel $[10,11]$. A modeling study of wild-type and mutant KCNQ3 channels suggested a critical stabilizing interaction between the PH and the S4-selectivity filter that is responsible for rearrangements of the P-loop architecture induced by the presence of a hydroxyl-containing residue at the 315 position, "unlocking" the channels into a conductive conformation [12]. Tyr270 is predicted

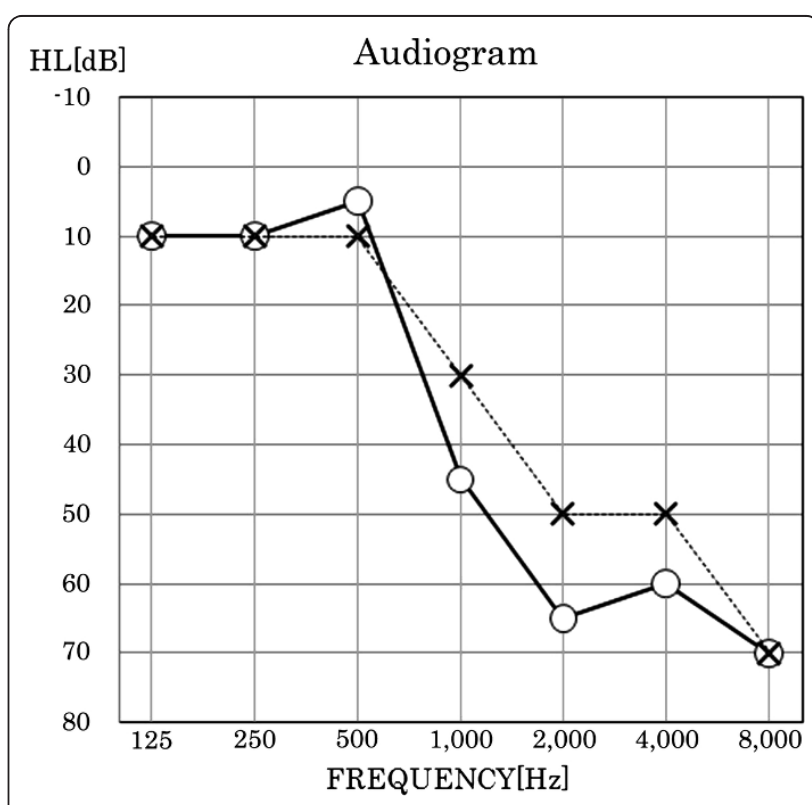

Figure 2 An audiogram from the patient with Tyr270His. Open circles connected by the solid line are the hearing levels for the right ear. Crosses connected by the dotted line are the hearing levels for the left ear. 
to be the N-terminal residue of the $\mathrm{PH}$. Available mammalian KCNQ4 pore-region sequences are identical, and those of non-mammals (e.g., birds, fruit flies, tunicates, and nematodes; Figure $3 \mathrm{~B}$ ) are very similar to mammalian sequences. In addition, the $\mathrm{PH}$ and P-loop sequences of non-KCNQ4 Kv channels (e.g., Kv2.1 from Homo sapiens is identical in 14/24 amino acid residues, Kv1.2 from Rattus norvegicus in $11 / 24$, and KvAP, a prokaryotic Kv channel from Aeropyrum pernix in 13/ 24) are somewhat conserved, suggesting that the pore residues, including Tyr270, are necessary for the proper functioning of the channel (Figure 3C).

Given the patient's clinical data and that the Tyr270His mutation is within the highly conserved pore region, we built structural models of wild-type KCNQ4 and the Tyr270His pore regions in an attempt to understand how the mutation affected $\mathrm{K}^{+}$transport and, consequently, the patient's hearing. To identify a template crystal structure, we searched the Protein Data Bank using Gapped BLAST [13] and PDBsum [14], to obtain a three-dimensional structure of a KCNQ4 homolog. Among the channels with available three-dimensional structures, the Kv1.2 sequence was most similar to that of KCNQ4 (27.5\% identity for the transmembrane sequence Ser32 to Thr417). Consequently, structural models for the transmembrane regions (Tyr80 to Gln329) of wild-type KCNQ4 and Tyr270His were built based on the template given by the crystal structure of Kv1.2 using SWISS-MODEL http://swissmodel.expasy. org/[15-17]. The reliabilities of the models were evaluated using Verify_3D http://nihserver.mbi.ucla.edu/Verify_3D/[18,19], and were found to be trustworthy (see Additional file 1: Figure S1).

The $\alpha$-carbon tracing of the KCNQ4 model was visualized using Chimera http://plato.cgl.ucsf.edu/chimera/ [20]. Tyr270, at the N-terminus of $\mathrm{PH}$, was found at the same position as the corresponding Kv1.2 residue (Ile361) (Figure 4A). We therefore assessed the electrostatic characteristics of the two models to determine if a positively charged His270 side chain might be responsible for the patient's hearing loss.

The ribbon model of wild-type KCNQ4 with the electrostatic surface potential superimposed, suggests that the side chain of Tyr270 (colored white in Figure 4B) should be electrostatically neutral. In contrast, for Tyr270His, the side chain of His270 (colored blue in Figure $4 \mathrm{C}$ ) is predicted to retain at least a partial positive charge, which reflects a standard $p \mathrm{~K}_{\mathrm{a}}$ value (6.5) for Histidine. Moreover, His270 is surrounded by the negatively charged residues Asp272, Asp266, Asp262, Glu260, and the polar residues Ser269, Ser268, and Ser265, which are capable of hydrogen bonding (all the aforementioned residues are within $10 \AA$ of His 270 , Figure 4D). In hemoglobin (PDB ID: 2hhb), the side-chain $p \mathrm{~K}_{\mathrm{a}}$ of His97, which is located in the loop domain between helix 3 and helix 4 and surrounded by negatively charged residues of Asp94 (located contiguous to the Cterminus of helix 3) and Asp99 (N-terminus of helix 4) within $5 \AA$ distance, is shifted to an abnormally high value $\left(\mathrm{pK}_{\mathrm{a}}=7.8\right)$ [21]. For these reasons, the side chain of His270 may also have a $p \mathrm{~K}$ value that is greater than the standard value, and, therefore, it was predicted that more than half of the histidine side chains at position 270 carry a positive charge near physiological $\mathrm{pH}$ (Figure 4C).

The alteration of the electrostatic surface potential of a single helical residue in the pore region might affect the structural stability of the channel as a consequence of an alteration in the helix dipole moment. Substantial dipole moments with positive and negative unit charges at the $\mathrm{N}$ - and $\mathrm{C}$-terminals, respectively, of $\alpha$-helices are often found [21]. Comparison of three Kv channel structures suggests that the dipoles of the transmembrane helices orient the helices and are responsible, at least in part, for the structure of the pore [22-24]. The electrically neutral Tyr270, which, as noted above, is at the Nterminus of the $\mathrm{PH}$, is located within $9 \AA$ of Ala263, the S5 C-terminus (Figure 4B, C). The presence of a positively charged His adjacent to, or at the N-terminal of, an $\alpha$-helix can destabilize the helix dipole [25]. Replacement of Tyr270 with His may therefore increase the dipole moment of the $\mathrm{PH}$.

Alternatively, or in conjunction with the destabilization effect, His270 may impede $\mathrm{K}^{+}$transport. The electrostatic repulsion between a positively charged His270 and $\mathrm{K}^{+}$would be stronger than that between the electrically neutral Tyr270 and $\mathrm{K}^{+}$. In the Tyr270His model, the distance between His270 and the center pore of the channel was $\sim 20 \AA$ (Figure 4E, F). This distance is small enough to affect the electrostatic interaction between two charged molecules in a non-polar environment (e.g., the interior of a membrane protein) because the electrostatic force is strong and affects charges separated by 500-1800 ̊ [26-28]. Additionally, the dielectric constantsin the interior of a protein and in a lipid membrane are assumed to be 5 and 2, respectively, whereas that for bulk water is $\sim 80$ at room temperature [29]. The electrostatic potential energy for two charges in protein or lipid membrane interiors is therefore between 16 and 40 times greater than that in water. Consequently, the long-range electrostatic repulsive force between the positively charged His 270 and a $\mathrm{K}^{+}$might possibly impede $\mathrm{K}^{+}$passage through the channel [30], whereas the force on $\mathrm{K}^{+}$would be smaller in the extracellular region.

Several mutations (e.g., Leu274His and Trp276Ser) in the KCNQ4 PH have been correlated with hearing loss [8]. The clinical features, congenital, progressive high- 


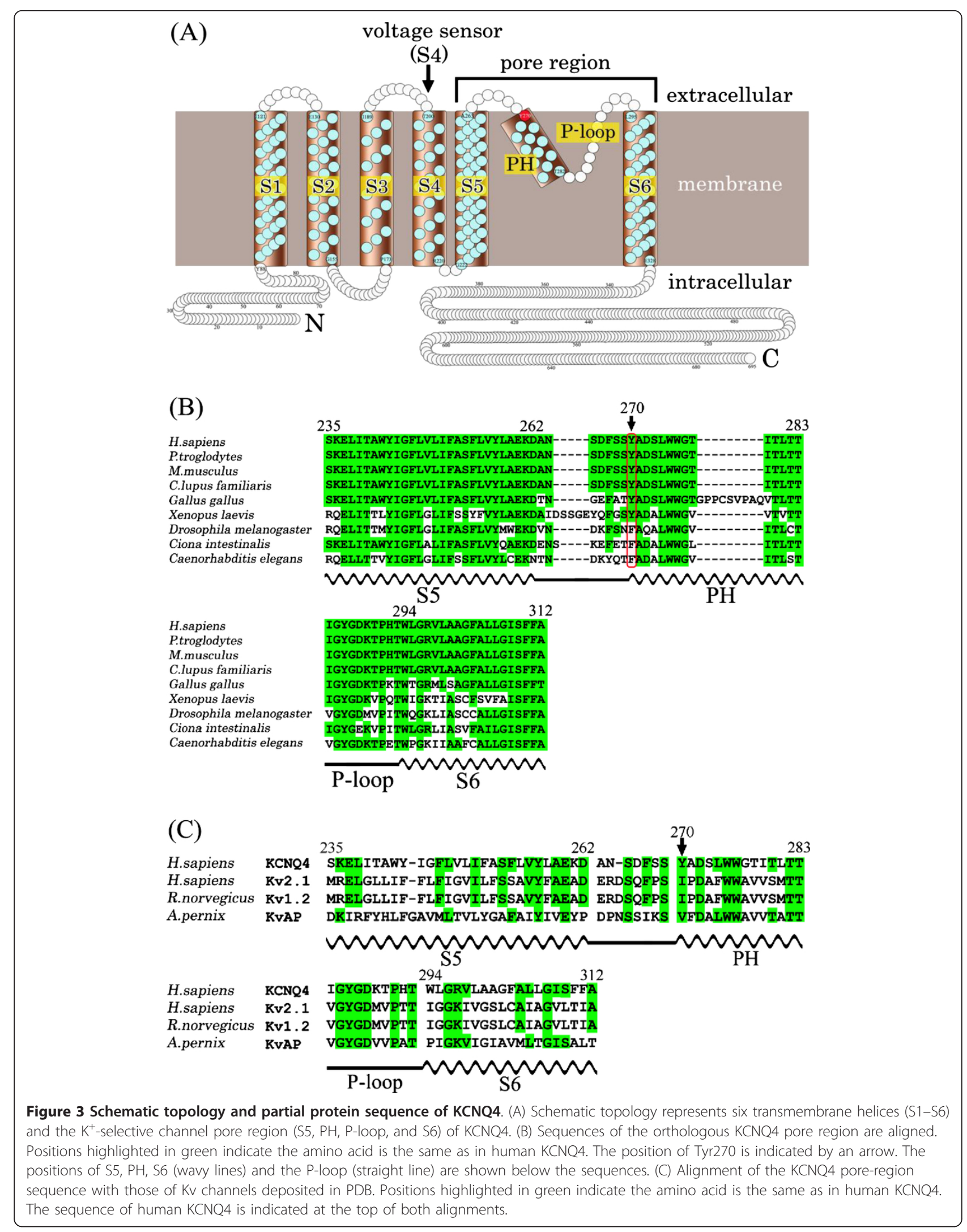



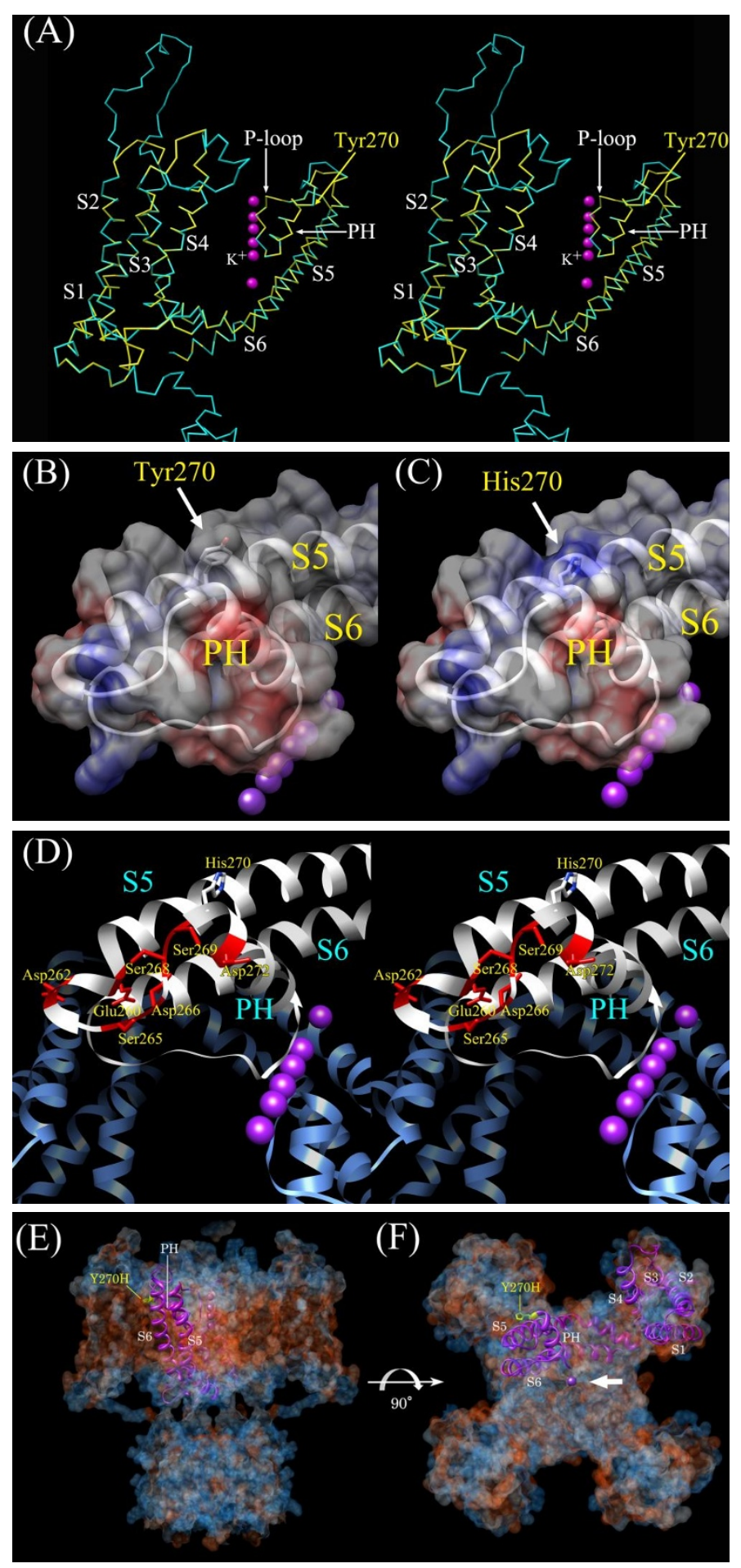

Figure 4 Structural models of KCNQ4 and Tyr270His. (A) Stereoview of the KCNQ4 $\alpha$-carbon frame model (yellow) superimposed onto that of Kv1.2 (cyan). The position of Tyr270 is indicated with a yellow arrow. The $\alpha$-helices, the PH, and the P-loop are also identified. (B) Part of the wild-type KCNQ4 model, and (C) the Tyr270His model overlaid with their corresponding electrostatic surface potentials. The side chains of Tyr270 (B) and His270 (C) are indicated by arrows. Negatively and positively charged residues are depicted respectively in red and blue in the electrostatic potential surface representations. (D) Stereoview of a portion of the ribbon model of the Tyr270His pore region. Residues that are negatively charged or that can form a hydrogen bond and surround His270 are identified and shown in red. (E, F) The Tyr270His ribbon model was superimposed onto (E) a horizontal view of the plasma membrane and (F) an extracellular view with the four rotational axes of the Kv1.2 crystal structure shown as hydrophobicity surface. Hydrophobic and hydrophilic residues are depicted respectively in red and blue in the hydrophobicity surface representations. His 270 is shown in yellow. (A-F) $\mathrm{K}^{+}$in the central pore is shown as a purple sphere and indicated by an arrow in (F). 
frequency hearing loss without substantial loss of speech recognition during the first decade of life, were the same for the patients with the aforementioned mutated KCNQ4s and the patient with Tyr270His [8,31]. Interestingly, ectopic expression of the Trp276Ser mutant and wild-type KCNQ4 in a cultured cell line caused a reduction in the channel current, whereas expression of only the mutant caused impaired trafficking of the protein to the cellular membrane [32]. The similar clinical symptoms and locations of the mutations within KCNQ4 support the proposal that Tyr270His is a pathogenic mutant associated with progressive SNHL. Further physiological and three-dimensional structural characterization of Tyr270His may identify which of our working hypotheses, structural distortion of the channel caused by a change in the $\mathrm{PH}$ dipole moment or electrostatic impediment of $\mathrm{K}^{+}$transport, or both, caused hearing loss in the patient, and provide insight into how to reverse hearing loss caused by $\mathrm{KCNQ} 4$ mutants.

\section{Conclusion}

In summary, we identified a new KCNQ4 mutant, in which Tyr270 was replaced with His in a patient with progressive SNHL. Structural models of wild-type and Tyr270His pore regions revealed that His270 caused an alteration in the electrostatic surface potential of the $\mathrm{PH}$, which, in turn, suggests that the molecular pathology of Tyr270His might cause the hearing loss in two ways. First, the increased dipole moment of the $\mathrm{PH}$ caused by the positively charged His 270 might destabilize the PH structure, as well as that of the pore region. In parallel, the electrostatic repulsive force between His270 and $\mathrm{K}^{+}$might impede $\mathrm{K}^{+}$transport through the channel.

\section{Methods}

\section{Subjects}

The Research Ethics Committee of the National Tokyo Medical Center approved this study. Subjects with bilateral sensorineural hearing loss were recruited by the National Tokyo Medical Center and collaborating hospitals. Informed, written consent was obtained from the patients and their parents before removing genetic material. Medical histories and physical examinations were acquired for 127 patients whose hearing loss was not a syndromic symptom or a consequence of environmental factors (e.g., perinatal complication, meningitis, prenatal or postnatal drug ototoxicity, or acoustic trauma). Before sequencing the pore regions of the patients' $K C N Q 4$, their mitochondrial DNA sequences (m.1555A > G or m.3243A > G) and GJB2 were determined to be normal (mutations in these genomic regions are known to be the most frequent causes of hereditary hearing loss $[33,34])$. Each patient's hearing level was examined by pure-tone audiometry. Hearing loss was classified according to pure-tone average values at $0.5,1,2$, and $4 \mathrm{kHz}$ http://hereditaryhearingloss.org/.

\section{Genetic analysis}

Genomic DNA was extracted from blood samples using the reagents of the Gentra Puregene Blood kit (QIAGEN, Hamburg, Germany). For polymerase-chain-reaction (PCR) amplification of $K C N Q 4$ exons 5,6 , and 7, the primer sets 5'-GAGATGGGGGACCTTTATCC-3' and 5'AGCCCTACAAAGACCCTCAC-3'; 5'-GACCAGTCCTG CCTGTAACC-3' and 5'-AACTGAGCAGGAGGCAA CTC-3'; and 5'-ACCCTTGCAGCCTCTTACTG-3' and 5'-CTGCTCCTAGGGCTTCTTCC-3; respectively, were used in conjunction with AmpliTaq Gold DNA polymerase (Applied Biosystems, Foster City, CA) and a PC-818 Program Temp Control System (ASTEC, Shizuoka, Japan). The PCR program was: $95^{\circ} \mathrm{C}$ for $4 \mathrm{~min} ; 30$ three-step cycles of $95^{\circ} \mathrm{C}$ for $30 \mathrm{~s}, 59.4^{\circ} \mathrm{C}$ for $30 \mathrm{~s}$, and $72^{\circ} \mathrm{C}$ for $45 \mathrm{~s}$; and then $72^{\circ} \mathrm{C}$ for $5 \mathrm{~min}$. Primer sets for all $K C N Q 4$ exons (Additional file 2: Table S1) were used to characterize the DNA encoding Tyr270His. These exons were amplified using PrimeSTAR HS DNA polymerase (Takara Bio, Shiga, Japan). All other PCR-amplification conditions were the same as mentioned above. PCR products were sequenced using an ABI 3730 DNA sequence analyzer and the reagents of the ABI Prism Big Dye Terminator Cycle Sequencing kit (Applied Biosystems). Characterization of the sequences was undertaken using SeqScape software v2.6 (Applied Biosystems) and DNASIS Pro (Hitachisoft, Tokyo, Japan), with the KCNQ4 sequence (NG_008139, NCBI Build37.1) as the reference. Control DNA was obtained from 96 Japanese subjects with normal hearing.

\section{Molecular modeling of KCNQ4}

To find a voltage-gated $\mathrm{K}^{+}$channel with a sequence homologous to that of KCNQ4 that could be used as the structural template for the modeling exercise, we searched the Protein Data Bank using Gapped BLAST [13] and PDBsum (European Bioinformatics Institute: http://www.ebi.ac.uk/pdbsum/. The transmembrane helices and the voltage sensor and pore regions of KCNQ4 and Tyr270His were then modeled using SWISS-MODEL [15-17], with the crystal structure of Kv1.2 (PDB ID: 3LUT, chain B) as the template [11]. Both KCNQ4 models were built using the automatic modeling mode and default parameters. The qualities of the models were evaluated using the Verify_3D Structure Evaluation Server (see Additional file 2: Table S1) $[18,19]$. Finally, the models were each superimposed onto Kv1.2 using Chimera [20] to visualize the modeled $\alpha$-carbon frames and ribbon models, with the electrostatic surface potentials of KCNQ4 or Tyr270His overlaid. 


\section{Additional material}

Additional file 1: Figure S1. The quality of the models was assessed by the Verify-3D program. The vertical axis indicates the average 3D-1D score for residues in 21-residue sliding window, the centre of which is at the sequence position indicated by the horizontal axis. Initial models of KCNQ4 exhibited better quality than those of the Kv1.2 template (Cys327 to Thr716). Nearly $100 \%$ of the scores were positive and most of these were higher than 0.1. Thus, we took the predicted KCNQ4 structure to be trustworthy.

Additional file 2: Table S1. The primer sets for KCNQ4. The number of each primer set indicates the target exons of KCNQ4. Each primer specific for a given genomic sequence (upper case) is conjugated with either a universal forward M13 (lower case) or a reverse M13pUC (lower case).

\section{Abbreviations}

DFNA2: Nonsyndromic autosomal dominant sensorineural deafness type 2; KCNQ4: Potassium voltage-gated channel; KQT-like subfamily: Member 4; Kv: Voltage-gated potassium channel; PCR: Polymerase chain reaction; SNHL: Sensorineural hearing loss.

\section{Acknowledgements}

This study was supported by a Health Science Research Grant (H16kankakuki-006 to TM) from the Ministry of Health, Labor, and Welfare of Japan, a Grant-in-Aid for Clinical Research to TM from the National Hospital Organization, and by a Grant-in-Aid for Scientific Research (19592001 to TM) from the Ministry of Education, Culture, Sports, Science and Technology of Japan.

\section{Author details}

${ }^{1}$ Laboratory of Auditory Disorders, National Institute of Sensory Organs, National Tokyo Medical Center, Tokyo, Japan. ${ }^{2}$ Department of Integrated Science in Physics and Biology, College of Humanities and Science, Nihon University, Tokyo, Japan. ${ }^{3}$ Department of Otolaryngology, National Sendai Medical Center, Miyagi, Japan.

\section{Authors' contributions}

$\mathrm{KN}$ was involved in the design of the study, performed the experiments and data analysis, and drafted the manuscript. HM was involved in the conception of the study and polished the manuscript. HK was involved in the conception of the study and gave considerations about structural information. SH provided information about the patient with sensorineural hearing loss. TM conceived the study, and participated in its coordination, design, data analysis and production of the manuscript. All authors read and approved the final manuscript.

\section{Competing interests}

The authors declare that they have no competing interests.

Received: 28 November 2011 Accepted: 15 March 2012 Published: 15 March 2012

\section{References}

1. Smith RJ, Bale JF Jr, White KR: Sensorineural hearing loss in children. Lancet 2005, 365:879-890.

2. Morton CC, Nance WE: Newborn hearing screening-a silent revolution. $N$ Engl J Med 2006, 354:2151-2164.

3. Kochhar A, Hildebrand MS, Smith RJ: Clinical aspects of hereditary hearing loss. Genet Med 2007, 9:393-408.

4. Coucke P, Van Camp G, Djoyodiharjo B, Smith SD, Frants RR, Padberg GW, Darby JK, Huizing EH, Cremers CW, Kimberling WJ: Linkage of autosomal dominant hearing loss to the short arm of chromosome 1 in two families. N Engl J Med 1994, 331:425-431.

5. Kubisch C, Schroeder BC, Friedrich T, Lütjohann B, El-Amraoui A, Marlin S, Petit C, Jentsch TJ: KCNQ4, a novel potassium channel expressed in sensory outer hair cells, is mutated in dominant deafness. Cell 1999, 96:437-446.
6. Robbins J: KCNQ potassium channels: physiology, pathophysiology, and pharmacology. Pharmacol Ther 2001, 90:1-19.

7. Topsakal V, Pennings RJ, te Brinke $H$, Hamel B, Huygen PL, Kremer $H$, Cremers CW: Phenotype determination guides swift genotyping of a DFNA2/KCNQ4 family with a hot spot mutation (W276S). Otol Neurotol 2005, 26:52-58

8. Van Hauwe P, Coucke PJ, Ensink RJ, Huygen P, Cremers CW, Van Camp G: Mutations in the KCNQ4 $\mathrm{K}^{+}$channel gene, responsible for autosomal dominant hearing loss, cluster in the channel pore region. Am J Med Genet 2000, 93:184-187.

9. Coucke PJ, Van Hauwe P, Kelley PM, Kunst H, Schatteman I, Van Velzen D, Meyers J, Ensink RJ, Verstreken M, Declau F, Marres H, Kastury K, Bhasin S, McGuirt WT, Smith RJ, Cremers CW, Van de Heyning P, Willems PJ, Smith SD, Van Camp G: Mutations in the KCNQ4 gene are responsible for autosomal dominant deafness in four DFNA2 families. Hum Mol Genet 1999, 8:1321-1328.

10. Chen X, Wang Q, Ni F, Ma J: Structure of the full-length Shaker potassium channel Kv1.2 by normal-mode-based X-ray crystallographic refinement. Proc Natl Acad Sci USA 2010, 107:11352-11357.

11. Long SB, Tao X, Campbell EB, MacKinnon R: Atomic structure of a voltagedependent $\mathrm{K}+$ channel in a lipid membrane-like environment. Nature 2007, 450:376-382.

12. Zaika O, Hernandez CC, Bal M, Tolstykh GP, Shapiro MS: Determinants within the turret and pore-loop domains of KCNQ3 $\mathrm{K}+$ channels governing functional activity. Biophys J 2008, 95:5121-5137.

13. Altschul SF, Madden TL, Schäffer AA, Zhang J, Zhang Z, Miller W, Lipman DJ: Gapped BLAST and PSI-BLAST: a new generation of protein database search programs. Nucleic Acids Res 1997, 25:3389-3402.

14. Laskowski RA: Enhancing the functional annotation of PDB structures in PDBsum using key figures extracted from the literature. Bioinformatics 2007, 23:1824-1827.

15. Arnold K, Bordoli L, Kopp J, Schwede T: The SWISS-MODEL Workspace: A web-based environment for protein structure homology modeling. Bioinformatics 2006, 22:195-201.

16. Kiefer F, Arnold K, Künzli M, Bordoli L, Schwede T: The SWISS-MODEL Repository and associated resources. Nucleic Acids Res 2009, 37:387-392.

17. Peitsch MC: Protein modeling by E-mail. Bio/Technology 1995, 13:658-660.

18. Bowie JU, Lüthy $R$, Eisenberg D: A method to identify protein sequences that fold into a known three-dimensional structure. Science 1991, 253:164-170.

19. Lüthy R, Bowie JU, Eisenberg D: Assessment of protein models with three-dimensional profiles. Nature 1992, 356:83-85.

20. Pettersen EF, Goddard TD, Huang CC, Couch GS, Greenblatt DM, Meng EC, Ferrin TE: UCSF Chimera-A Visualization System for Exploratory Research and Analysis. J Comput Chem 2004, 25:1605-1612

21. Hol WG: Effects of the a-helix dipole upon the functioning and structure of proteins and peptides. Adv Biophys 1985, 19:133-165.

22. Doyle DA, Morais Cabral J, Pfuetzner RA, Kuo A, Gulbis JM, Cohen SL, Chait BT, Mackinnon R: The structure of the potassium channel: molecular basis of $\mathrm{K}^{+}$conduction and selectivity. Science 1998, 280:69-77.

23. Roux B, Mackinnon R: The cavity and pore helices in the KcsA K $\mathrm{K}^{+}$ channel: electrostatic stabilization of monovalent cations. Science 1999, 285:100-102

24. Dutzler R, Campbell EB, Cadene M, Chait BT, MacKinnon R: X-ray structure of a CIC chloride channel at $3.0 \mathrm{~A}$ reveals the molecular basis of anion selectivity. Nature 2002, 415:287-294.

25. Armstrong KM, Baldwin RL: Charged histidine affects alpha-helix stability at all positions in the helix by interacting with the backbone charges. 1993, 90:11337-11340.

26. $\mathrm{Xu} \mathrm{XH}$, Yeung ES: Long-range electrostatic trapping of single-protein molecules at a liquid-solid interface. Science 1998, 281:1650-1653.

27. Kang SH, Yeung ES: Dynamics of single-protein molecules at a liquid/ solid interface: implications in capillary electrophoresis and chromatography. Anal Chem 2002, 74:6334-6339.

28. Larsen AE, Grier DG: Like-charge attractions in metastable colloidal crystallites. Nature 1995, 385:230-233.

29. Callenberg KM, Choudhary OP, de Forest GL, Gohara DW, Baker NA, Grabe M: APBSmem: a graphical interface for electrostatic calculations at the membrane. PLOS ONE 2010, 5, pii: e12722. 
30. Li N, Tang H, Gai H, Dong X, Wang Q, Yeung ES: Determination of protein surface excess on a liquid/solid interface by single-molecule counting. Anal Bioanalytical Chem 2009, 394:1879-1885.

31. De Leenheer EM, Huygen PL, Coucke PJ, Admiraal RJ, van Camp G, Cremers CW: Longitudinal and cross-sectional phenotype analysis in a new, large Dutch DFNA2/KCNQ4 family. Ann Otol Rhinol Laryngol 2002, 111:267-274.

32. Kim HJ, Lv P, Sihn CR, Yamoah EN: Cellular and molecular mechanisms of autosomal dominant form of progressive hearing loss, DFNA2. J Biol Chem 2011, 286:1517-1527.

33. Matsunaga T, Hirota E, Bito S, Niimi S, Usami S: Clinical course of hearing and language development in GJB2 and non-GJB2 deafness following habilitation with hearing aids. Audiol Neuro 2006, 11:59-68.

34. Matsunaga T: Value of genetic testing in determination of therapy for auditory disorders. Keio J Med 2009, 58:216-222.

doi:10.1186/1756-0500-5-145

Cite this article as: Namba et al: In silico modeling of the pore region of a KCNQ4 missense mutant from a patient with hearing loss. BMC Research Notes 2012 5:145.

\section{Submit your next manuscript to BioMed Central} and take full advantage of:

- Convenient online submission

- Thorough peer review

- No space constraints or color figure charges

- Immediate publication on acceptance

- Inclusion in PubMed, CAS, Scopus and Google Scholar

- Research which is freely available for redistribution

Submit your manuscript at www.biomedcentral.com/submit 